\title{
Bedeutung von Sport und Ernährung für die körperliche Leistungsfähigkeit und Gesundheit - Ein systematischer Review
}

\author{
Reinhard Saller¹, Paolo M. Suter2, Christine Römer-Lüthi³, Christof Mannhart", Reto Brignoli5, Remy Meier ${ }^{6}$ \\ 1 UniversitätsSpital Zürich, Institut für Naturheilkunde, Zürich, Schweiz; 2 UniversitätsSpital Zürich, Abteilung für Hypertonie, Zürich, Schweiz; \\ 3 Berner Fachhochschule Gesundheit, Bern, Schweiz; 4 Eidgenössische Hochschule für Sport Magglingen EHSM, Magglingen, Schweiz; \\ 5 Facharzt für Pharmakologie, Rüti, Schweiz; ${ }^{6}$ Medizinische Universitätsklinik, Abteilung für Gastroenterologie, Liestal, Schweiz
}

\begin{abstract}
port ist die willkürliche Schaffung von Aufgaben, Problemen oder Konflikten, die vorwiegend mit körperlichen Mitteln gelöst werden. Die Lösungen können beliebig wiederholt, verbessert und geübt werden, und die Handlungsergebnisse führen nicht
\end{abstract}

\section{Was ist Sport?}

unmittelbar zu materiellen Veränderungen. Sport ist auch Spass, Freude, Ausgleich, körperliche Betätigung, Teamgeist, an seine Grenzen gehen, den Körper spüren und vieles andere.

Der Brockhaus (17. Auflage, 1973) definiert Sport wie folgt: „... Sammelbezeichnung für die an spielerischer Selbstentfaltung (Spiel) und am Leistungsstreben orientierten Formen menschlicher Betätigung, die der körperlichen und geistigen Beweglichkeit dienen, besonders auf dem Gebiet der Leibesübungen. Diese Tätigkeiten, die in den meisten Fällen um ihrer selbst willen und aus Freude an der Überwindung von Schwierigkeiten ausgeübt werden, sind gewöhnlich regelgebunden und werden im freiwilligen Wettkampf und in eigens dafür bestimmten Organisationsformen gepflegt. Die spielerische Bewegung zur Selbststeigerung ohne Wettkampf-streben entfaltet sich zumeist individuell und unorganisiert."

„Sport wird somit konstituiert durch

- motorische Aktivitäten, die auf den

Erwerb und das Verbessern spezifischer koordinativer und konditioneller Fähigkeiten abzielen,

- in der Regel motorische Handlungen, die im Erreichen bzw. Übertref-
Hintergrund: Viele Menschen bewegen sich heute zu wenig und ernähren sich unausgewogen. Dies hat negative Auswirkungen auf das Wohlbefinden, die körperliche Leistungsfähigkeit und ganz allgemein auf die Gesundheit. Zielsetzung: Erstellung eines systematischen Review zur Bedeutung von Sport und Ernährung für die Gesundheit. Methoden: Systematische Analyse und Bewertung von Humanstudien (prospektive Doppelblindstudien, epidemiologische und retrospektive Studien, kurzfristige biochemische/hämatologische Studien ( Surrogate Markers»)) der letzten 10 Jahre aus den gängigen elektronischen Datenbanken sowie der Angaben von Standardwerken und publizierten Monographien. Ergebnisse und Schlussfolgerungen: Sportförderung stellt eine wichtige präventiv-medizinische Massnahme dar. Grundlage für die benötigte körperliche Bewegung ist beispielsweise die Bewegungspyramide. Zur Verbesserung sportlicher Leistung muss ein Training nach dem Prinzip der schrittweise steigenden Belastung aufgebaut sein. Die Ernährung von SportlerInnen sollte - wie die Ernährung von Personen, die keinen Sport betreiben - grundsätzlich auf der Lebensmittelpyramide basieren. Manche Sportlerlnnen benötigen zusätzliche Nährstoffe. Körperliche Leistungsfähigkeit nimmt zwar mit dem Alter ab, die Leistungsfähigkeit kann aber im Alter durch Training erhalten und verbessert werden. Die positive Wirkung des Trainings auf Blutfettwerte und besonders auf Herz und Kreislauf ist abhängig von der Trainingsintensität. Schwitzen ist ein zentraler Regulationsmechanismus der durch Training oder Sport erhöhten Körpertemperatur. Mit Schweiss und Verdunstung werden ausser Wasser auch Elektrolyte ausgeschieden, die wieder zu ersetzen sind. Viele Sportlerlnnen fragen sich, ob sie mit Nahrungsergänzungsmitteln oder anderen - legalen! - Wirkstoffen ihre Leistung verbessern können. Zurzeit gibt es wenige Daten über dazu geeignete Substanzen.

Schlüsselwörter: Sport, Ernährung, Leistungsfähigkeit, Gesundheit

\section{A Systematic Review of the Significance of Sport and Diet for Physical Performance and Health}

Background: Many people exercise too little nowadays and have a diet that lacks balance. This has negative effects on wellbeing, physical performance and on general health Objective: To conduct a systematic review of the significance of sport and diet for health. Methods: Systematic analysis and evaluation of human studies - including prospective double-blind studies, epidemiological and retrospective studies, short term biochemical and haematological studies with surrogate markers - performed in the last 10 years and found in major electronic data bases, coupled to information in standard works and published monographs. Results and Conclusion: The promotion of sport represents a significant measure in terms of preventive medicine. For example, the exercise pyramid counts as a basis for required physical activity. In order to be able to improve sporting performance, a training régime must be built up in accordance with the principle of increasing exposure to physical effort by stages. The diet of athletes should - as for that of people who take no part in sport - be based on the food pyramid. Many athletes require additional nutrients. Though physical capability declines with age, it can be maintained and improved in old age by means of training. The positive effect of training on cholesterol levels and particularly on the heart and circulation is dependent on the intensity of training. Perspiration is a central regulatory mechanism for body temperature increased by training or sport. By means of perspiration and evaporation, electrolytes are also eliminated along with water. These have to be replaced. Many athletes confront the dilemma as to whether they could improve their performance using dietary supplements or other (legal!) substances. Currently, there is little data available on suitable substances.

Keywords: Sport, diet, physical performance, health 
fen weitgehend künstlich gesteckter Ziele ihren Sinn haben,

- einen charakteristischen und verbindlichen Handlungskodex, mit dem sportliche Aktivitäten hinsichtlich der Abläufe, der Organisationsstrukturen und der Handlungsnormen geregelt sind ....

- die künstliche Erzeugung einer Ebene, auf der motorische Aktivitäten von Aspekten des produktiven Nutzhandelns freigesetzt sind und sich überwiegend konsequenzlos, also spielerisch vollziehen können..."

Soweit die Theorie. Über die Hälfte der Menschheit bewegt sich zu wenig; physische Inaktivität soll gemäss WHO jährlich weltweit 1,9 Millionen vorzeitige Todesfälle verursachen, hiervon ca. 600'000 in Europa [1].

Die quergestreifte Muskulatur bildet beim Menschen etwa 55\% der Körpermasse - wie bei den meisten anderen Säugetieren auch. Die Muskulatur ist nicht nur für die Bewegungen, sondern auch für die Produktion von Wärme und den gesamten Stoffwechsel zentral. Das Leistungspotential und Anpassungsvermögen der querge- streiften Muskulatur wird beim Sport stark gefordert. Wer die Fähigkeiten der quergestreiften Muskulatur voll ausschöpfen will, kann dies heute nur durch bessere Kenntnisse der genetischen, biochemischen und biophysikalischen Grundlagen erreichen.

Als Grundlage für die benötigte körperliche Bewegung gilt beispielsweise die vom Österreichischen Verein zur Förderung von Ernährungsinformationen vorgeschlagene Bewegungspyramide (Abbildung 1). Die Ernährung von Sportlerinnen und Sportlern sollte - wie auch die Ernährung von Personen, die keinen Sport betreiben grundsätzlich auf der Lebensmittelpyramide basieren (Abbildung 2). Die Lebensmittelpyramide ist ein Leitfaden zur Auswahl der Lebensmittel; sie gilt für gesunde Erwachsene, die höchstens moderat körperlich aktiv sind. Sportlerinnen und Sportler, die intensiv Sport betreiben, benötigen zusätzliche Nährstoffe. Eine Übersicht dazu bieten beispielsweise Infoblätter der ETH Zürich (www.sfsn.ethz.ch, Swiss Forum for Sport Nutrition).

\section{Leistungssteigerung durch Training}

Das Grundprinzip jedes Trainings lautet: Belastung $\Rightarrow$ Ermüdung $\Rightarrow$ Erholung $\Longrightarrow$ Leistungssteigerung durch biologische Anpassung. Der optimale Wechsel zwischen Belastung und Erholung ist Voraussetzung für einen Leistungszuwachs des Organismus und für ein erfolgreiches Training (Abbildung 3). Ist die Erholungsphase zu lang, sinkt die Leistungsfähigkeit allmählich wieder auf das Ausgangsniveau ab.

Der Zuwachs an Muskelkraft oder Ausdauer ist eine biologische Anpassung des Organismus, damit er zukünftige Anforderungen besser bewältigen kann. Jedes Training führt wegen der Ausschöpfung der Energiereserven dazu, dass das Leistungsniveau vorübergehend sinkt (Ermüdung). In der Erholungsphase regeneriert sich der Organismus, und das Ausgangsniveau der Leistungsfähigkeit wird wieder erreicht. Ist die Erholungsphase von optimaler Dauer, kommt es zu einer Erhöhung der Leistungsfähigkeit über das Ausgangsniveau hinaus (Mehrausgleich - Überkompensation).

\section{Die Bewegungspyramide für Kinder und Erwachsene}

Kinder: $60+$ bewegte Minuten täglich

Erwachsene: $30+$ bewegte Minuten täglich

Sitzende Freizeit

KInder: Je weniger, desto besser

Erwachsene: ]e weniger, desto besset

Kraftsport

Kinder: 23 xpro Woche, nach Lust und Laune Erwachsene: 2-3x pro Woche, mind. 10 Minuten

\section{Ausdauersport}

Erwachsene: $3-5 x$ pro woche,

mindestens 20 Minuten

Alltagsbewegung

Erwachsene: Liaglich

mindesters 30 Minuter

Ausdauer- und Alltagsbewegung

Kinder: zusammen mindestens

1 Stunde täglich

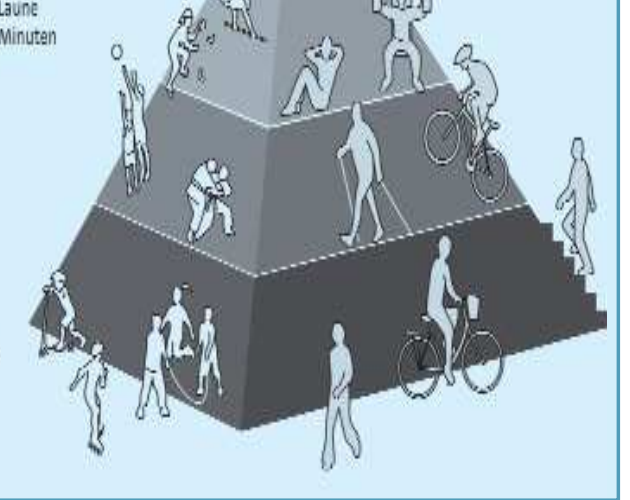

Abb. 1. Die Bewegungspyramide (2006, Österreichischer Verein zur Förderung von Ernährungsinformationen, www.forum-ernaehrung.at).

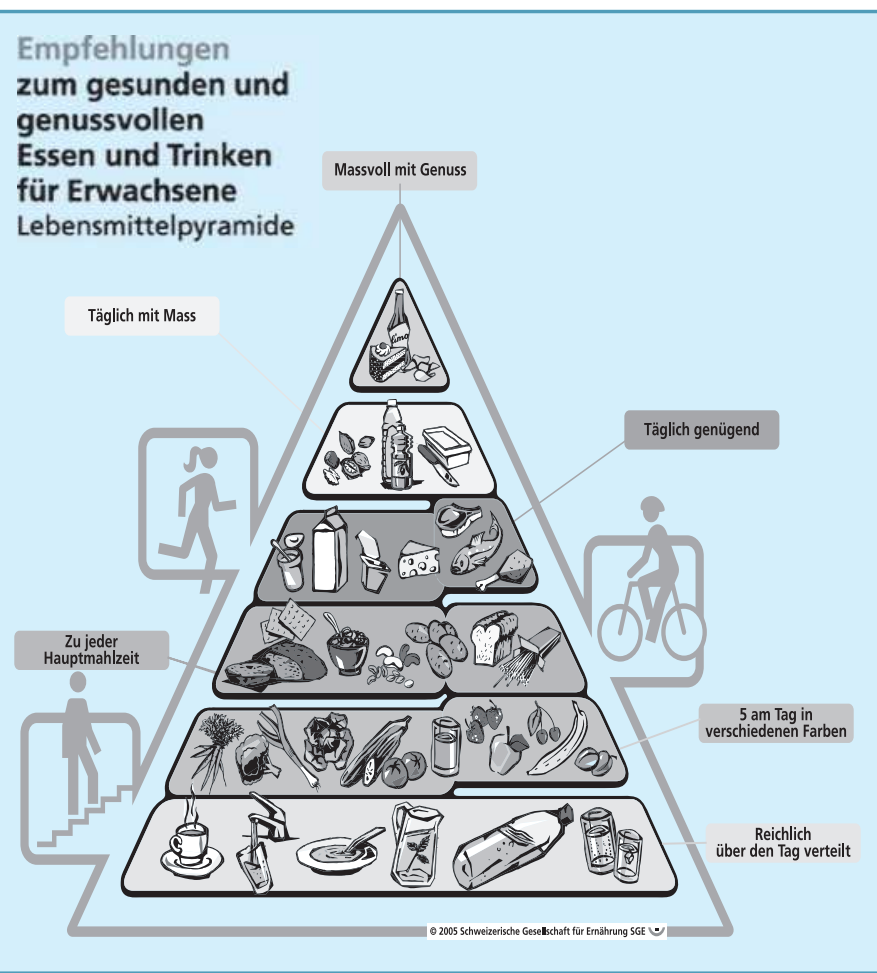

Abb. 2. Schweizer Lebensmittelpyramide zur Lebensmittelauswahl (2005 Schweizerische Gesellschaft für Ernährung SGE, www.sge-ssn.ch). 


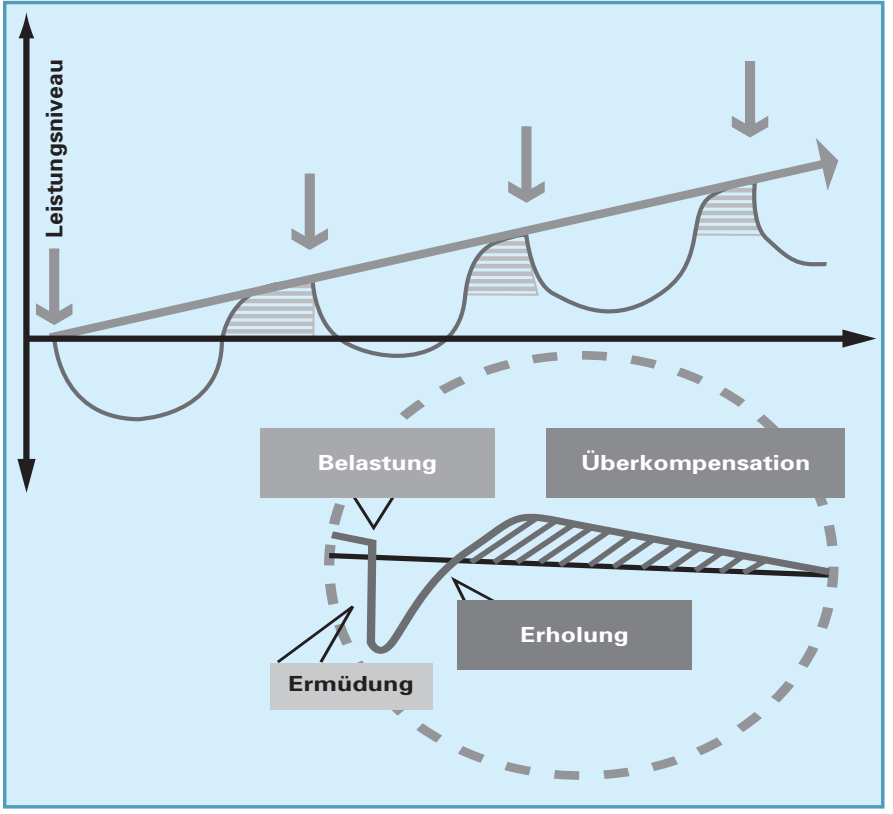

Abb. 3. Leistungssteigerung durch biologische Anpassung.

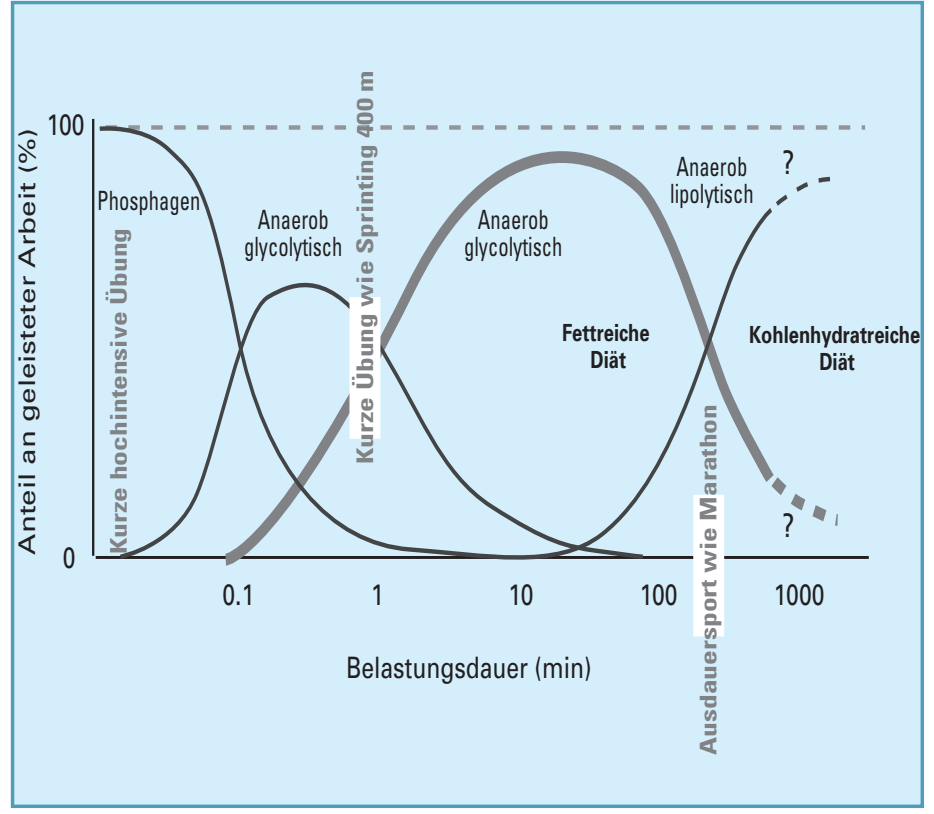

Abb. 4. Energiebereitstellung in Abhängigkeit von der Belastungsdauer [6]
Damit die Leistung kontinuierlich zunimmt, müssen die Trainingsreize nach dem Prinzip der steigenden Belastung schrittweise erhöht werden. Man kann also durchaus sagen, dass der Trainingsprozess selbst einen Druck nach ständiger Leistungssteigerung erzeugt. Andererseits hat die stetig wachsende und unmittelbar erfahrbare Leistung auch einen stark motivierenden Effekt, so dass das Training fortgesetzt wird.

\section{Der Skelettmuskel}

Die Skelettmuskulatur besteht aus spezialisierten Zellen, welche die Muskelfasern bilden. Das Zellinnere besteht vor allem aus Eiweissfäden, die sich zusammenziehen können (Myofibrillen). Die Zellen selbst sind zwischen 0,1 und $15 \mathrm{~cm}$ lang, zylindrisch geformt und enthalten oft mehr als 100 Zellkerne (pro Zelle!); diese Zellkerne sind ganz an den Rand der Zelle gedrängt, nahe dem ca. $10 \mathrm{~nm}$ dicken Sarkolemm, einer elastischen Bindegewebshautschicht [2].

Die fadenförmigen Myofibrillen, die mit ca. $85 \%$ den Hauptbestandteil des Muskelfaservolumens ausmachen, haben einen Durchmesser von ca. 1-2 $\mu \mathrm{m}$ und sind in Längsrichtung der Faser angeordnet. Die Myofibrillen sind vom
Sarkoplasma umspült, in dem sich unter anderem Ionen, Gase, Energiespeicherstoffe wie Glykogen (in Körnchenform) und Fetttröpfchen, RNS in allen Formen, Aminosäuren, Proteine, Enzyme und weitere Zellorganellen befinden. Zu den wichtigsten Zellorganellen gehören neben den Zellkernen, die die genetischen Informationen in Form der DNS tragen, auch die Mitochondrien. In diesen „Kraftwerken der Zelle” findet die von Sauerstoff abhängige (aerobe), oxidative Energiegewinnung statt. Auch das sarkoplasmatische Retikulum ist als Calcium-Speicher massgeblich an der Muskelkontraktion beteiligt.

Die Muskelfasern werden in zwei Grundtypen unterteilt: Die dunklen Fasern vom Typ I (oder ST) haben eine langsame Kontraktionsrate und eine hohe oxidative Kapazität, aber ein geringeres anaerobes - sauerstoffloses Potential zur Energieverarbeitung als die hell gefärbten „schnellen” Fasern vom Typ II (oder FT). Die Muskeln von Marathonläufern enthalten ca. $80 \%$ Fasern vom Typ I, da diese für die Ausdauer entscheidend sind [4]. Die Fasern vom Typ II verkürzen sich dagegen rund zehnmal schneller als die langsamen Fasern. Die Typ-I-Fasern sind besser ansprechbar für Insulin; die Insulinsensibilität kann sich aber, speziell bei Muskelläsionen, vorüber- gehend verschlechtern [5].

Beeinflusst wird die Kontraktionsgeschwindigkeit der Muskelfasern durch die unterschiedliche Geschwindigkeit, mit der Adenosintriphosphat (ATP) gespalten und verbraucht wird. ATP ist der universelle Energielieferant aller Zellen. Typ-I-Fasern regenerieren ATP langsamer über den Stoffwechselweg, der Sauerstoff verbraucht; daher eignen sie sich vor allem für Ausdauersport wie Langstreckenlaufen, Radfahren oder Schwimmen. Schnelle Typ-II-Fasern können kurzfristig über einen anaeroben Stoffwechselweg ATP gewinnen, ermüden hingegen rascher; sie sind daher für kurzdauernde Leistungen wie beim Gewichtheben oder Sprinten zuständig (Abbildung 4). Grundsätzlich kann die Muskulatur längerfristig nur so viel Energie verbrauchen, wie Sauerstoff zur Verfügung steht. Das maximale Vermögen zur Sauerstoffaufnahme ist ein wichtiger leistungsbestimmender Faktor im Sport, vor allem beim Ausdauersport.

Eine Muskelfaser ist mit dicht gepackten Myofibrillen gefüllt. Diese bestehen der Länge nach aus einer Folge von Sarkomeren, die jeweils von zwei Z-Scheiben begrenzt werden. Jede Myofibrille ist von Längskanälen des sarkoplasmatischen Reticulums (Longitudinal- oder L-Tubuli) umsponnen. Quer zu diesen durchziehen in 
regelmässsigen Intervallen tubuläre Plasmalemma-Einstülpungen die Faser und bilden das erregungsleitende Netzwerk der Transversal- oder T-Tubuli. An den Berührungsflächen zwischen T- und L-Tubuli liegen Membrankontakte für die Übertragung der Erregung.

\section{Genetische Faktoren}

Was ist wichtiger bei sportlichen Leistungen, die genetische Ausstattung der Athleten oder das Training? Diese Frage wird immer wieder gestellt. Fest steht, dass sowohl eine optimale genetische Ausstattung als auch intensives Training nötig sind, um sportliche Spitzenleistungen erzielen zu können.

Eine Zusammenfassung der aktuellen wissenschaftlichen Literatur, welche die sportlichen Leistungen bei jungen Zwillingsbrüdern untersucht, kommt zu folgendem Schluss: Etwa 25 $-40 \%$ der maximalen Sauerstoffaufnahmekapazität sind erblich bedingt der Rest ist aktivitätsbedingt, also vom Trainingsgrad abhängig [7].

Die Genathlete-Studie ist eine FallVergleichsstudie zwischen herausragenden Ausdauerathleten $(\mathrm{n}=296)$ und „Normalpersonen” $(n=289)$ [8]. Man fand signifikante Übereinstimmungen zwischen der Leistungsfähigkeit der Athleten und einzelnen Merkmalen in ihren Genen - beispielsweise Genabschnitten, die für die Energiegewinnung durch Fettverbrennung zuständig sind. Die genaue Bedeutung dieser Befunde ist noch unklar und widerspricht teilweise den Befunden anderer Studien [9].

Marathonläufer afrikanischen Ursprungs verfügen im Durchschnitt ebenfalls über eine grössere Ausdauer und eine grössere oxidative Kapazität als Läufer kaukasischen Ursprungs, was ebenfalls auf eine genetische Komponente hinweist [10].

Hinweis auf einen genetischen „Vorteil” erfolgreicher Sportler ist auch der Wachstumsfaktor Myostatin. Mutationen im Myostatin-Gen führen zu einer $\mathrm{Zu}$ nahme der Muskelmasse. Man kennt Sportlerinnen mit gesteigertem Muskelaufbau und verlangsamtem Muskelabbau, bei denen solche Mutationen des Myostatin-Gens nachgewiesen werden konnten [11]. Auch der Wachstumsfaktor IGF-1 (Insulin-like growth factor) beeinflusst die Muskulatur. Mäuse mit einem implantierten IGF-1-Gen verfügen nicht nur über $20-50 \%$ mehr Muskulatur, sondern sie behalten im Alter ihre Fähigkeit zur Regeneration der Muskeln, was normalerweise nur bei jüngeren Tieren vorkommt.

\section{Körperliche Leistungs- fähigkeit im Alter}

Die körperliche Leistungsfähigkeit nimmt mit dem Alter grundsätzlich ab. Die durch Training gesteigerte Leistungsfähigkeit bleibt aber auch im Alter erhalten bzw. kann durch Training verbessert werden (Abbildung 5) [12]. Eine geringe körperliche Leistungsfähigkeit ist mit einer erhöhten allgemeinen Sterblichkeit verknüpft, und auch das Risiko für Herz-Kreislauf-Krankheiten ist erhöht [13,14].

Es konnte nachgewiesen werden, dass sowohl ein intensives Training als auch „brisk walking” (rasches Gehen) zu einer signifikanten Abnahme der Herz-Kreislauf-Ereignisse bei Frauen führen. Die positive Wirkung der körperlichen Bewegung auf die Blutfettwerte und auf andere Faktoren, welche die „Fitness” des Herz-Kreislauf-Systems anzeigen, ist abhängig von der Intensität des Trainings [15,16,17].

Eine Analyse von 27 kontrollierten Studien, welche die Wirkung von körperlichem Training bei Patienten mit
Typ-2-Diabetes untersuchten, zeigte folgende Resultate: Nach mehr als zwölf Wochen Training verbesserte sich der HbA1c-Wert um 0,8 $\pm 0,3 \%$, speziell bei schlecht kontrolliertem Diabetes. Der HbA1c-Wert zeigt den Verlauf der Blutzuckerwerte über längere Zeit an. Auch andere Faktoren wie Blutdruck und Blutfettwerte verbesserten sich. Am besten schnitten Patienten ab, die ein Ausdauer- und Krafttraining kombinierten; ein ausschliessliches Ausdauertraining erbrachte bessere Ergebnisse als ein ausschliessliches Krafttraining [18].

In diesem Zusammenhang muss erwähnt werden, dass die Kreislaufbelastung beim Sport auch ein Risiko darstellen kann. Beispielsweise ist das Risiko für einen plötzlichen Herztod unter körperlicher Belastung höher als in Ruhe. Besonders hoch ist das Risiko, wenn jemand nach einer Zeit ohne vorhergehendes Training plötzlich mit Sport anfängt, und bei starker Trainingsintensität [19]. So sollten vor allem ältere Menschen (> 50 Jahre), die nach einer längeren Bewegungspause mit einem sportlichen Training beginnen wollen, ihre Leistungsfähigkeit vorher vom Arzt abklären lassen.

\section{Wasser- und Wärmeregulation}

Muskeln arbeiten nicht sehr effizient. Sie setzen nur etwa einen Viertel der Energie in mechanische Arbeit um, der

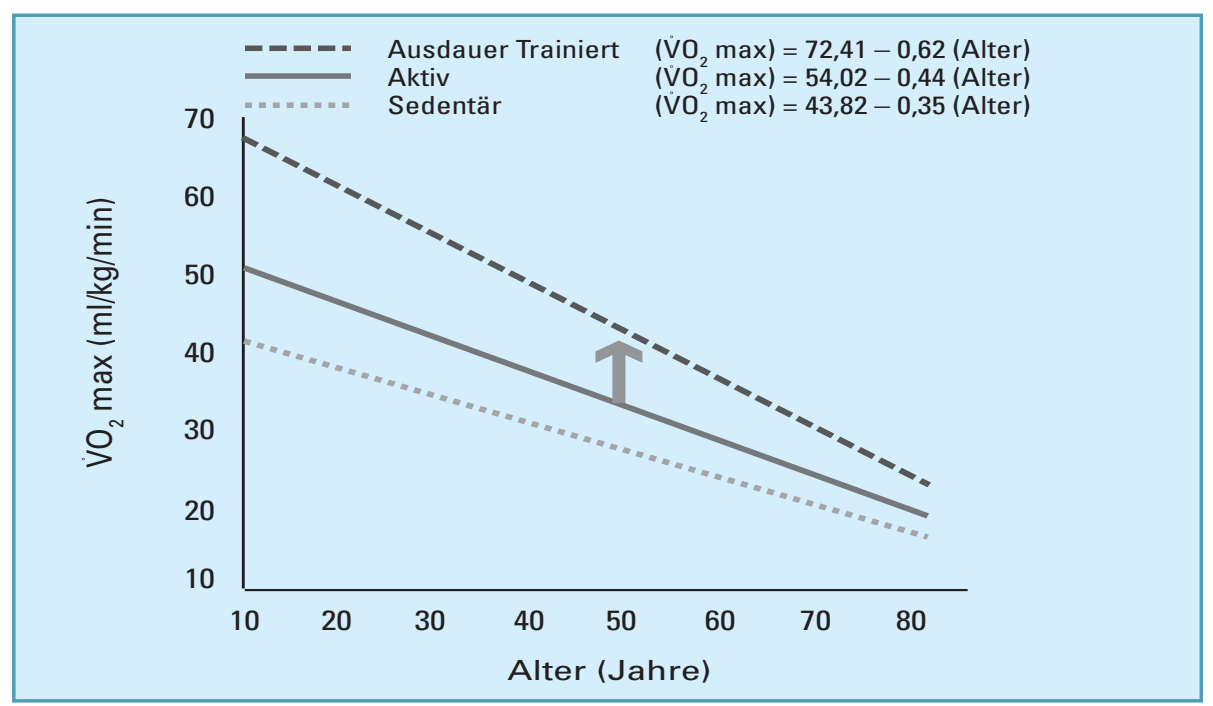

Abb. 5. Zusammenhang zwischen Training, Leistungsfähigkeit und Alter [12]. 


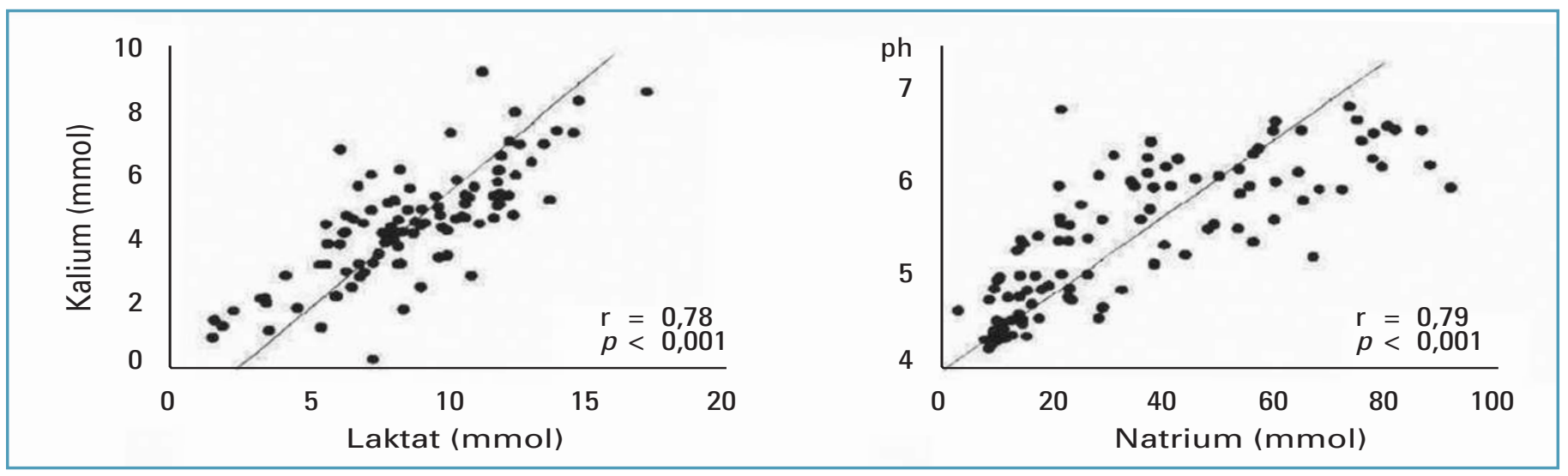

Abb. 6. Korrelation zwischen Kalium- und Laktatgehalt im Schweiss (links) sowie pH-Wert und Natriumgehalt des Schweisses (rechts).

Rest geht als Wärme verloren. Die überschüssige Wärme führt zum Schwitzen: Dieser zentrale Regulationsmechanismus hält die Körpertemperatur konstant. Mit dem Schweiss und der Verdunstung werden Wasser und Elektrolyte ausgeschieden.

Wassermangel ist derjenige Faktor, der bei körperlicher Anstrengung als erster die Leistung begrenzt. Ein Wassermangel (Dehydratation) von ein bis zwei Prozent der Körpermasse kann die physische Leistungsfähigkeit messbar vermindern. Bei einer etwa $70 \mathrm{~kg}$ schweren Person ist dieser Verlust bereits nach etwa einer Stunde sportlicher Betätigung gegeben (durchschnittlicher Schweissverlust etwa ein Liter pro Stunde). Mit zunehmender körperlicher Leistung nimmt die Bedeutung der Hydratation für die Wärmeregulation zu.

Besonders bei Marathonläufern ist Hyponatriämie ein häufiges zusätzliches Problem („Marathon Maladies”) [20]. In einer Studie wurde festgestellt, dass durchschnittlich 13\% der Teilnehmer von Marathonläufen (22\% der Frauen und $8 \%$ der Männer) eine Hyponatriämie haben (Natrium unter $135 \mathrm{mmol} / \mathrm{l})$. Risikofaktoren für eine Hyponatriämie sind eine Gewichtszunahme während des Laufs, lange Laufzeit von über vier Stunden und ein niedriges Körpergewicht (Body-MassIndex unter 20). Hauptursache für eine Gewichtszunahme ist eine exzessive Flüssigkeitszufuhr von über drei Litern pro Stunde. Die Autoren der Studie raten, bereits im Training sowie vor und nach dem Rennen Gewichts- kontrollen durchzuführen. Andere Studien haben auch die Einnahme von nicht-steroidalen Antiphlogistika als Risikofaktor für eine Hyponatriämie ausmachen können.

Zwei Mechanismen kommen theoretisch als Auslöser für die Hyponatriämie in Frage: Der Verlust von Natriumchlorid (Kochsalz) durch massives Schwitzen resp. verstärkte Urinausscheidung, oder die „Verdünnungshyponatriämie” bei übermässiger $\mathrm{Zu}$ fuhr hypotoner Flüssigkeit [21,22]. Es wird empfohlen, dass Ausdauersportler während des Trainings isotonische Getränke zu sich nehmen, um den Wasser- und Elektrolytverlust auszugleichen (ca. 0,4 bis 0,8 Liter pro Stunde Belastung). Besonders geeignet ist eine Natrium-Kalium-Elektrolyt-Mischung mit Glukose, Saccharose, Fruktose und Maltodextrin; diese Mischung muss individuell angepasst werden.

Bezüglich Wasserhaushalt und Ausdauersport gelten folgende Grundsätze [23]:

- Bei 70 kg Körpergewicht besteht der Mensch aus etwa 42 Liter Wasser.

- Das Wasser, das beim Training verloren geht, wird zu 85-95\% über Schweiss abgegeben.

- Wenn der Wasserverlust über $2 \%$ des Körpergewichts beträgt, kommt es zur Leistungseinbusse.

- Eine „Überwässerung” (Hyperhydratation) bietet keine Vorteile in Bezug auf die Wärmeregulierung.

- Bei einer Hyperhydratation besteht das Risiko einer Hyponatriämie (besonders bei Frauen und bei Menschen, die über 65 Jahre alt sind).
- Eine Dehydratation erhöht das Risiko für Erschöpfung, Hitzschlag, Muskelkrämpfe und Rhabdomyolyse (Muskelschädigung, die zu akutem Nierenversagen führen kann).

\section{Schwitzen und Elektrolyte}

Während eines intensiven Trainings werden grössere Mengen Elektrolyte mit dem Schweiss ausgeschieden [24, 25]. Probanden, die auf einer 400-mTartanbahn zehn Kilometer laufen, verlieren durchschnittlich $20 \mathrm{mg}$ Calcium, $5 \mathrm{mg}$ Magnesium, $200 \mathrm{mg} \mathrm{Ka-}$ lium und $800 \mathrm{mg}$ Natrium mit dem Schweiss [26]. Wie viele Elektrolyte (Natrium, Kalium, Calcium, Clorid) mit dem Schweiss ausgeschieden werden, ist unabhängig von der individuellen Fitness [27]. Zwischen dem pH-Wert und dem Natriumgehalt des Schweisses besteht eine gute Korrelation, ebenso wie zwischen dem Kalium- und dem Laktatgehalt (Abbildung 6). Die Variationen zwischen den einzelnen Probanden sind beachtlich [28].

\section{Magnesium}

Die Aufnahme von Magnesium mit der Nahrung liegt häufig unter der empfohlenen Tagesmenge. Gleichzeitig ist die Ausscheidung von Magnesium bei Hitze und bei körperlicher Anstrengung erhöht. Es stellt sich die Frage, ob eine Supplementierung von Magnesium bei Sportlern sinnvoll ist.

In Studien wurde festgestellt, dass bei Sportlern, die in der Hitze acht Stunden Fahrrad gefahren sind, die Ausscheidung von Magnesium mit dem Harn und mit dem Schweiss erhöht ist 
(Ausscheidung mit dem Harn $+21 \%$ : $131,5 \mathrm{mg} / \mathrm{d}$ vs. $108,6 \mathrm{mg} / \mathrm{d}$ in Ruhe; Ausscheidung mit dem Schweiss: 15,2$17,8 \mathrm{mg} \mathrm{Mg} / \mathrm{d}$ ). Parallel dazu wurde eine Reduktion der Magnesium-Plasmaspiegel beschrieben [29]. Saunabäder bewirken höhere Magnesiumund Calcium-Konzentrationen im Schweiss als die Belastung durch Training. Allerdings ist es so, dass mit zunehmendem Schweissfluss die Magnesium-Konzentration im Schweiss abnimmt. Eine epidemiologische Studie weist darauf hin, dass zwischen klimatischer Hitzebelastung, Magnesiumverlust und dem vermehrten Auftreten von tödlichen Herzinfarkten und Diabetes ein Zusammenhang bestehen könnte [30].

In einer Studie mit künstlichem Magnesium-Entzug wurden bei den Probanden die Sauerstoffaufnahme, die Herzfrequenz und die Ausdauer bei submaximaler Belastung gemessen [31]. Trotz einer geringeren Ausdauer waren die Sauerstoffaufnahme und die Herzfrequenz in der Phase des Magnesium-Entzugs erhöht.

Die Mischung von Zink und Magnesium als Supplement soll bei Sportlern die Erholungsphase verkürzen, den Muskelaufbau fördern und die Kraft im Training steigern. Dies wurde in einer kleinen Studie bei knapp 30 Fussballspielern nachgewiesen; dieses Resultat bedarf aber noch weiterer Bestätigung [32].

Schlussfolgernd ist der Einfluss von Magnesiumsupplementen auf die Leistungsfähigkeit nicht klar. Generell gesehen dürfte eine Supplementierung mit Magnesium die Leistung nicht beeinflussen, solange der Magnesiumgehalt im Blut im Normbereich liegt. Bei einem versteckten oder sogar klinischen Magnesiummangel hingegen dürfte eine Supplementierung die Leistung verbessern [33,34].

\section{Zink}

Die empfohlene Tagesdosis Zink beträgt in der Altersgruppe von 19-30 Jahren $12 \mathrm{mg}$ bei Frauen und $15 \mathrm{mg}$ bei Männern. Bei Sportlern ist die Zinkaufnahme häufig zu gering [35].

In einer Studie mit künstlichem Zinkentzug wurden an verschiedenen Muskelgruppen der Knie und der
Schultern sowohl die maximale Leistung als auch die totale Leistung gemessen. Der induzierte Zinkmangel hatte zwar keinen Einfluss auf die maximale Leistung, aber es wurde eine signifikante Abnahme der totalen Muskelleistung festgestellt [36].

Bei Volleyballern konnte man während der Trainingsperiode eine progressive Zunahme der Zinkausscheidung im Harn und im Schweiss messen [37]. Auch bei japanischen Soldaten nahm während einer intensiven 24-wöchigen Trainingsperiode die Ausscheidung von Zink und Kupfer im Harn zu [38]. Nach intensivem Training kann beobachtet werden, dass die Zinkkonzentration im Blutplasma zunimmt, während die Konzentration in den Zellen abnimmt [39].

In einer Studie aus dem Jahr 2002 wurden die Zinkverluste bei Ausdauertraining wie folgt erfasst: Die Ausscheidung von Zink mit dem Harn steigt um 50-60\%; die Ausscheidung mit dem Schweiss erhöht sich während der Anpassungsphase von 0,8 auf $13,7 \mathrm{mg} / \mathrm{d}$, später beträgt sie $2,2 \mathrm{mg} / \mathrm{d}$ [40].

Schlussfolgernd ist der Einfluss von Zinksupplementen auf die Leistungsfähigkeit nicht klar. Generell gesehen dürfte eine Supplementierung mit Zink die Leistung nicht beeinflussen, solange der Zinkwert im Blut im Normbereich liegt. Bei einem klinischen Zinkmangel hingegen dürfte eine Supplementierung die Leistung verbessern.

\section{Eisen}

Die Eisenverluste mit dem Schweiss sind beim Sport gering. Ausdauersportarten verursachen jedoch klinisch relevante Blutverluste, beispielsweise durch ischämische Colitis (Minderdurchblutung des Darms), Magenerosionen und gastroösophagealen Reflux. Besonders bei Dauerbelastungen, wie etwa Marathonläufen, hat man bei $8-30 \%$ der Läufer versteckte Blutverluste im Stuhl festgestellt; bei Extremsportlern, die über 100 Meilen laufen, verlieren sogar $83 \%$ Blut mit dem Stuhl [41].

Besonders bei jungen Athletinnen hat der Eisenmangel grosse Bedeutung; Ursachen sind der erhöhte Bedarf, eine eisenarme Ernährung und Blut- verluste, etwa durch die Menstruation oder stressbedingte Blutungen. In einer Studie aus Australien hatten $51,1 \%$ der Athletinnen subklinisch abnormale Eisenwerte und 4,6\% hatten klinisch manifeste Störungen [42]. Diese Befunde traten bei Männern viel seltener auf. Eisenmangel führt zur Leistungsminderung und sollte deshalb korrigiert werden $[43,44]$.

Um das Risiko eines Eisenmangels zu minimieren, sollten Sportler, die sich vegetarisch ernähren, Sportler im Ausdauerbereich und allgemein weibliche Sporttreibende darauf achten, Lebensmittel zu sich zu nehmen, die reich an gut bioverfügbarem Eisen sind. Ausserdem sollte der Eisenstatus regelmässig kontrolliert werden. Eine Eisensupplementierung ist im Fall eines Eisenmangels angebracht. Auch in Fällen von tiefen Ferritingehalten im Blut ohne gleichzeitige Eisenmangelanämie könnte eine Supplementierung von Vorteil sein $[45,46,47]$.

In einer Studie mit künstlichem Eisenentzug erhielten 37 Athletinnen während acht Wochen täglich $135 \mathrm{mg}$ Eisen oder Placebo. Anschliessend wurde ihr Leistungsvermögen auf einer 15-km-Teststrecke geprüft. Am Ende der Behandlungszeit stellte man fest, dass die Teilnehmerinnen, die Eisen erhalten hatten, einen geringeren Energieverbrauch und tiefere Laktatspiegel hatten sowie weniger Sauerstoff verbrauchten [48].

Schlussfolgernd ist der Einfluss von Eisensupplementen auf die Leistungsfähigkeit nicht klar. Generell gesehen dürfte eine Supplementierung mit Eisen die Leistung nicht beeinflussen, solange die Eisenwerte im Blut im Normbereich liegen. Eine Supplementierung von Eisen ist beim Vorliegen eines Eisenmangels angebracht.

\section{Andere anorganische Mikronährstoffe Chrom}

Trotz anfänglich guten Resultaten ergaben neuere, gut kontrollierte Studien keinen Nutzen einer Chromsupplementierung bei gesunden Probanden, die während des Trainings für 4 bis 16 Wochen täglich 200-800 $\mu \mathrm{g}$ Chrom einnahmen [49,50]. In einer Zusammenfassung zum Thema aus 
Tab. 1. Wirkung anorganischer Mikronährstoffe auf die Leistungsfähigkeit

\begin{tabular}{|c|c|c|c|}
\hline Nährstoff & $\begin{array}{l}\text { Leistung bei Mangel an } \\
\text { entsprechendem Nährstoff }\end{array}$ & $\begin{array}{l}\text { Wirkung auf } \\
\text { Muskel-Aufbau }\end{array}$ & $\begin{array}{l}\text { Leistungsfördernde } \\
\text { Wirkung }\end{array}$ \\
\hline Zink & Reduziert & - & - \\
\hline Magnesium & Reduziert & - & - \\
\hline Eisen & Reduziert & Unwirksam & Unwirksam \\
\hline Vanadium & Keine Angaben & Unwirksam & Unwirksam \\
\hline Bor & Keine Angaben & Unwirksam & Unwirksam \\
\hline Chrom & Reduziert? & Unwirksam & Unwirksam \\
\hline Phosphat & Keine Angaben & Keine Angaben & Möglich? * \\
\hline Selen & Reduziert? & Keine Angaben & Unwirksam \\
\hline Mangan & Keine Angaben & Keine Angaben & Keine Angaben \\
\hline Kupfer & $?$ & Keine Angaben & Keine Angaben \\
\hline
\end{tabular}

dem Jahr 2003 kommen die Autoren zu folgendem Schluss: „Seit mehr als zehn Jahren werden Studien mit Chrom-Supplementierung durchgeführt; keine der Studien hat einen Hinweis darauf erbracht, dass eine ChromSupplementierung die körperliche Leistungsfähigkeit von gesunden Personen beeinflussen könnte, sogar wenn die Supplementierung zusammen mit einem Trainingsprogramm erfolgt [51].”

\section{Selen}

Wie auch bei anderen Antioxidantien liegen keine Beweise vor, dass Selen die Leistung verbessert. In einer doppelblinden Studie mit 24 Athleten, die ein zehnwöchiges Ausdauertraining absolvierten, bewirkte die Einnahme von täglich $180 \mu \mathrm{g}$ Selen keine Steigerung der Sauerstoffaufnahme [52].

\section{Vanadium}

Vanadium soll, ähnlich wie Chrom, die Insulinsensibilität steigern und dadurch den Glukose- und Eiweissstoffwechsel beeinflussen. Entsprechende Studien mit gesunden Probanden, die während des Trainings täglich 0,5 $\mathrm{mg} / \mathrm{kg}$ Vanadium einnahmen, zeigten jedoch keinen Nutzen bezüglich Muskelmasse oder Kraft [53,54].

\section{Bor}

Bor soll die Synthese von Testosteron fördern. Zwei Studien, in denen täglich 2,5 mg Bor eingenommen wurden, er- gaben jedoch keinen Nutzen in Bezug auf Muskelmasse oder Kraft [55,56]. Eine Supplementierung mit Bor soll zu tieferen Phosphorwerten und höheren Magnesiumspiegeln im Blut führen [57].

Eine Zusammenfassung über die Bedeutung anorganischer Mikronährstoffe für die Leistungsfähigkeit ist in Tabelle 1 dargestellt.

\section{Leistungsfördernde Hilfsmittel}

Leistungsfördernde (ergogene) Hilfsmittel und Wirkstoffe sollen die körperliche Leistungsfähigkeit verbessern, Muskelgewebe aufbauen und Energiereserven vergrössern. $\mathrm{Zu}$ diesem Thema gibt es ausführliche Übersichtsarbeiten (Tabelle 2) [58,59]. In einer wichtigen Arbeit von RichaRd KreIDER aus dem Jahr 2004 werden die Hilfsmittel - pharmakologische oder Nahrungsergänzungsmittel - aufgeteilt in jene, welche die Muskeln aufbauen (Kreatin, Hydroxymethylbutyrat), und jene, die eine Leistungssteigerung bewirken (Natriumphosphat, Natriumbikarbonat, Kreatin, Koffein). Nicht diskutiert werden hier Wirkstoffe, die in den Dopinglisten erscheinen und die im Wettkampf verboten sind (wie beispielsweise Erythropoietin, Anabolika, Ephedrin, Narkotika etc.).
In einer Metaanalyse aus dem Jahr 2003 wurden Studien untersucht, die zwischen 1967 und 2001 mit verschiedenen Supplementen durchgeführt worden waren [60]. Voraussetzungen für den Einschluss in die Analyse waren, dass die Studie mindestens drei Wochen gedauert hatte und die Probanden mindestens zweimal pro Woche trainiert hatten. Von 250 Supplementen wurden nur sechs in mehr als zwei Studien untersucht. Kreatin und Hydroxymethylbutyrat bewirkten eine signifikante Zunahme des Magergewichts um $0,36 \%$ resp. $0,28 \%$ pro Woche sowie der Muskelkraft um 1,1\% resp. 1,4\% pro Woche (Abbildungen 7/8). Chrom, Dehydroepiandrosteron, Androstenedion und Eiweisse hatten keine signifikante Wirkung.

\section{Natriumbikarbonat, Natriumcitrat (Puffersubstanzen)}

Sowohl die einmalige Anwendung von Natriumbikarbonat und Natriumcitrat (Puffersubstanzen) als auch die Anwendung von Natriumbikarbonat über mehrere Tage werden aufgrund des leistungsfödernden Potentials als A-Supplemente klassifiziert. Das bedeutet, dass ,aufgrund verschiedener, an gesunden, nicht mangelernährten, trainierten Menschen durchgeführter Studien bei adäquater Anwendung und Dosierung eine direkte (schnell eintretende) positive Leistungsbeeinflussung wahrscheinlich ist” (www.dopinginfo.ch). 
Tab. 2. Nahrungsergänzungsmittel zur Leistungssteigerung (nach 58)

\begin{tabular}{|c|c|c|c|}
\hline Physiologische Funktion & Evidenz $\mathrm{A}^{\circ}$ & Evidenz B ${ }^{\circ}$ & Evidenz C ${ }^{\circ}$ \\
\hline \multirow[t]{2}{*}{ Hydratation } & Isotone-Getränke & Hypotone-Getränke & \\
\hline & & Glycerol & \\
\hline \multirow[t]{3}{*}{ Steigerung der Ausdauer } & Kohlenhydrate & Arginin & Capsaicin \\
\hline & Zitronensäure & Koffein & \\
\hline & & Carnitin & \\
\hline \multirow[t]{4}{*}{ Steigerung der Muskelkraft } & Proteine & Glutamin & Arginin \\
\hline & BCAA * & & \\
\hline & Kreatin & & \\
\hline & Hydroxymethylbutyrat & & \\
\hline \multirow{3}{*}{$\begin{array}{l}\text { Vorbeugung von Muskel- und } \\
\text { Gelenkverletzungen und Ermüdung }\end{array}$} & Kohlenhydrate & Vitamine $\mathrm{C}$ und $\mathrm{E}$ & Glucosamin \\
\hline & Zitronensäure & Carotinoide, Flavonoide & Chondroitin \\
\hline & & Carnosin, Anserine & \\
\hline \multirow[t]{2}{*}{$\begin{array}{l}\text { Vorbeugung einer Abnahme } \\
\text { der Abwehrfunktionen }\end{array}$} & Kohlenhydrate & Vitamine $\mathrm{C}$ und $\mathrm{E}$ & \\
\hline & & Glutamin & \\
\hline \multicolumn{4}{|c|}{$\begin{array}{l}\text { * branched-chain amino acids, speziell die Aminosäuren Valin, Leucin und Is } \\
\text { Evidenzkategorien (nach Aoi et al.): } \\
\text { A: genügende wissenschaftliche Evidenz } \\
\text { B: suggestive, das heisst noch ungenügende wissenschaftliche Evidenz } \\
\text { C: noch ungenügende wissenschaftliche Evidenz, könnte aber effektiv sein }\end{array}$} \\
\hline
\end{tabular}

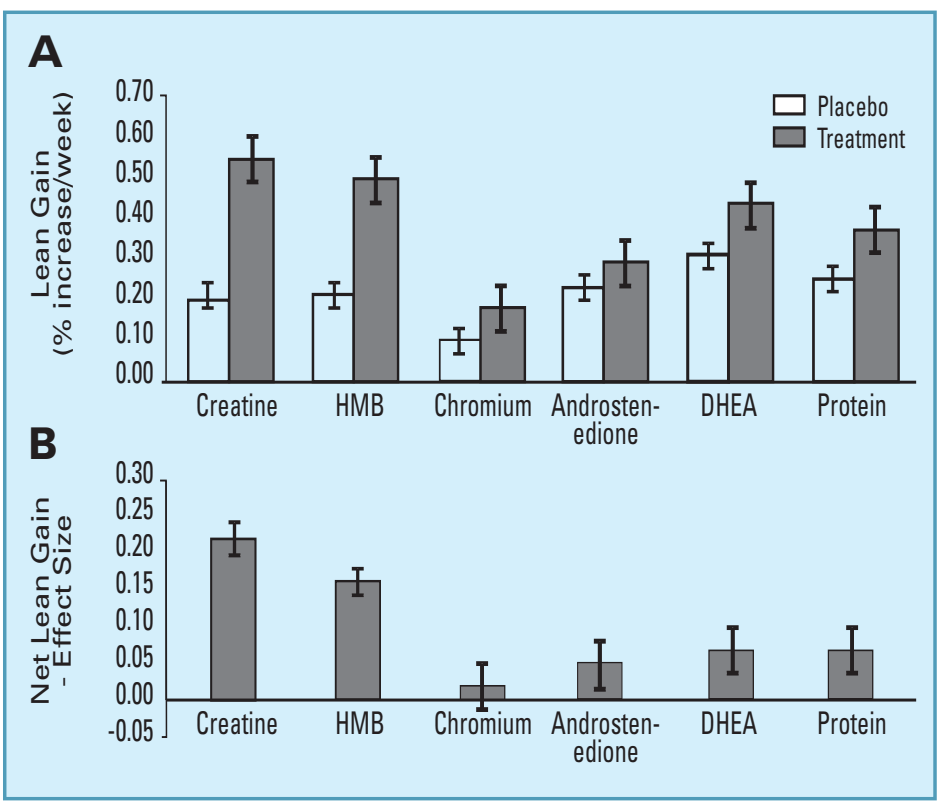

Abb. 7. Zunahme der Magermasse (ohne Fettgewebe) mit diversen Supplementen [60].

In neueren Studien wurde Bikarbonat über mehrere Tage eingenommen. Dabei verteilte man die tägliche Gesamtdosis auf ca. vier Einzeldosen über den Tag. Erstaunlicherweise scheint bei dieser mehrtägigen Einnahme die leistungsfördernde Wir-

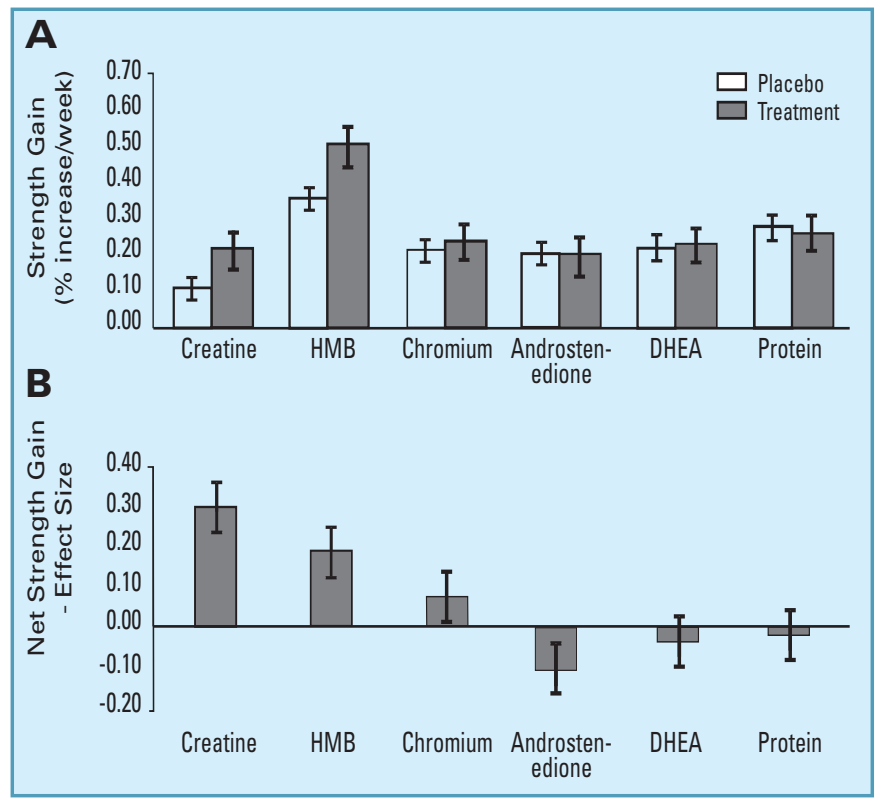

Abb. 8. Zunahme der Muskelkraft mit diversen Supplementen [60].
$27 \% \quad( \pm 20 \%) \quad z u$. In einer neueren Studie, die mit verschiedenen $\mathrm{Na}$ triumsalzen durchgeführt wurde, nahm die Zeit bis zur Erschöpfung ebenfalls um knapp 5\% zu [64].

Im Zusammenhang mit der Supplementierung von Natriumbikarbonat kung des Natriumbikarbonats bis zu zwei Tage nach Absetzen der Supplemente anzuhalten [61,62].

In einer Metaanalyse aus dem Jahr 1993 zeigten 19 von 35 Studien mit Bikarbonat positive Wirkungen [63]. Die Zeit bis zur Erschöpfung nahm um 


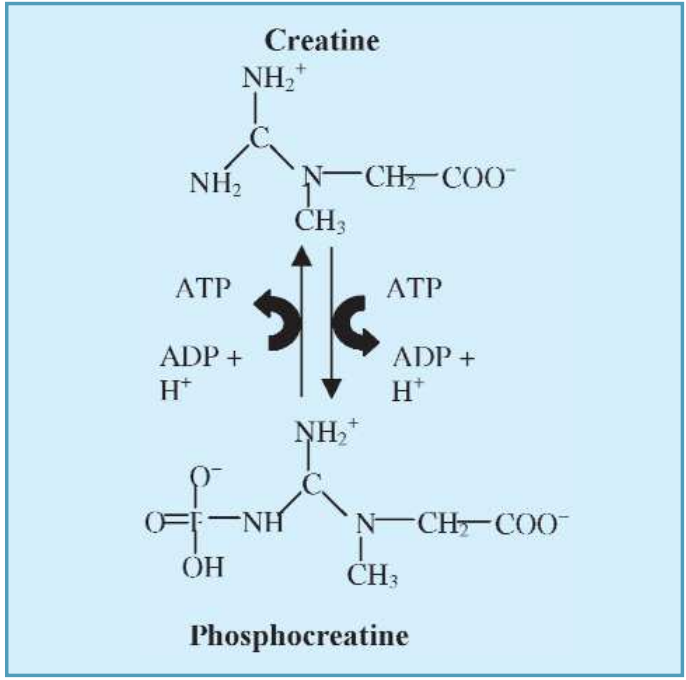

Abb. 9. Kreatin und Phosphokreatin.

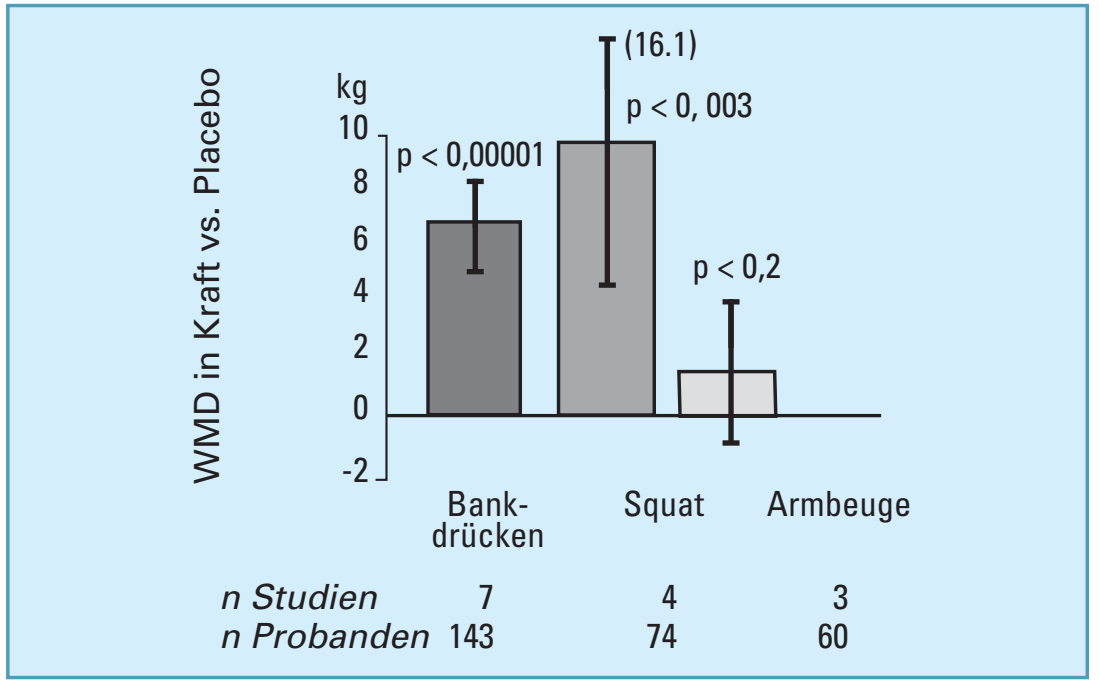

Abb. 10. Kreatin und Leistungssteigerung, Metaanalyse mit 16 Studien [66]. und Natriumcitrat sind allerdings häufig Magen-Darm-Probleme beschrieben worden. Andere Nebenwirkungen umfassen Störungen im Elektrolytstoffwechsel (Hypokaliämie, Hypernatriämie) sowie Störungen des peripheren und zentralen Nervensystems wie beispielsweise Gefühlsstörungen (Kribbeln, Parästhesien) und auch Krämpfe. Auch Herzrhythmusstörungen wurden unter der Einnahme von Puffersubstanzen beschrieben. Aus Gründen des nicht unbeträchtlichen Nebenwirkungspotentials müssen Puffersubstanzen also der Einteilung F zugeordnet werden: „Aufgrund der bisher an gesunden, nicht mangelernährten, trainierten Menschen durchgeführten Studien ist bei nicht adäquater Anwendung und Dosierung eine negative Leistungsbeeinflussung oder Nebenwirkungen nicht auszuschliessen" (www.dopinginfo.ch).

\section{Kreatin}

Kreatin ist ein körpereigener Stoff, der zur Energiebereitstellung in der Muskulatur gebraucht wird (Abbildung 9). Der tägliche Bedarf beträgt ungefähr zwei Gramm; ein Gramm wird vom Körper selbst produziert, der Rest wird über die Nahrung aufgenommen. Hering, Lachs, Rind- und Schweinefleisch enthalten am meisten Kreatin. Die zusätzliche Einnahme von Kreatin zeigt verschiedene positive Effekte: Man kann sich schneller erholen, die Belastbarkeit im Training ist grösser, die Kraft nimmt zu und Fett geht verloren.

Die übliche Dosierung beträgt täglich $0,3 \mathrm{~g}$ pro kg Körpergewicht während fünf bis sechs Tagen. In den ersten zwei Tagen wird in den Muskelzellen am meisten Kreatin gespeichert, anschliessend nimmt die Speicherung ab. Überschüssige Mengen an Kreatin werden über die Nieren ausgeschieden. Nach den ersten Tagen, die dazu dienen, den Organismus mit Kreatin „aufzufüllen” (Saturationsphase), reichen zwei Gramm Kreatin pro Tag aus, um das höhere Kreatinniveau im Körper zu erhalten [65].

In einer Metaanalyse, die 16 Studien umfasst, konnte unter der Supplementierung von Kreatin eine durchschnittliche Zunahme der Kraft beim Bankdrücken um rund $6,85 \mathrm{~kg}$ festgestellt werden [66]. Bei Armbeugen und bei der Fahrradergometrie ergab sich aber keine Kraftzunahme (Abbildung 10). Die Wirkung war besonders eindeutig bei jungen Männern, die Krafttraining machten. Bei älteren Personen und bei anderen körperlichen Leistungen konnte kein Nutzen nachgewiesen werden. Diese Resultate wurden in einer anderen Metaanalyse weitgehend bestätigt, wobei in dieser Studie auch festgestellt wurde, dass Kreatin bei Läufern und Schwimmern keinen Nutzen bringt [67]. Die Sicherheitsaspekte von Kreatin sind noch offen.

\section{Hydroxymethylbutyrat}

Hydroxymethylbutyrat (HMB) ist ein Stoffwechselprodukt der Aminosäure Leucin, das den Abbau von Eiweiss hemmen soll. HMB soll besonders untrainierten und älteren Personen am Anfang des Trainings zu grösserer Muskelmasse und Kraft verhelfen. Eine additive Wirkung mit Kreatin wurde ebenfalls beschrieben. Bei Athleten ist die Wirkung weniger eindeutig [58].

In den Jahren 2000-2005 wurden 13 Studien mit HMB durchgeführt, deren Resultate sind jedoch nicht eindeutig. Die Anzahl Teilnehmer ist in allen Studien zu gering, um ein falsch positives oder negatives Resultat ausschliessen zu können. Tendenziell ergibt sich eher eine Wirkung bei Kranken oder untrainierten Probanden, hingegen keine Wirkung bei Profisportlern (Tabelle 3). Eine neuere Metaanalyse zeigt eine signifikante Zunahme der Muskelkraft und des Magergewichts unter der Einnahme von HMB (NisSEN und SHARP). Für eine allgemeine Empfehlung ist die Beweislage allerdings ungenügend.

\section{Mittelkettige Triglyzeride}

Triglyzeride gelangen vom Darm ins Lymphgefässsystem und von dort gelangen sie als Chylomikronen in die Blutbahn. Im Blut werden die Triglyzeride $\mathrm{zu}$ ihrem jeweiligen Ziel transportiert; zum Beispiel ins Muskel- und Fettgewebe, um als Energiequelle gespeichert zu werden. 
Tab. 3. Klinische Studien 2000-2007 mit Hydroxymethylbutyrat HMB

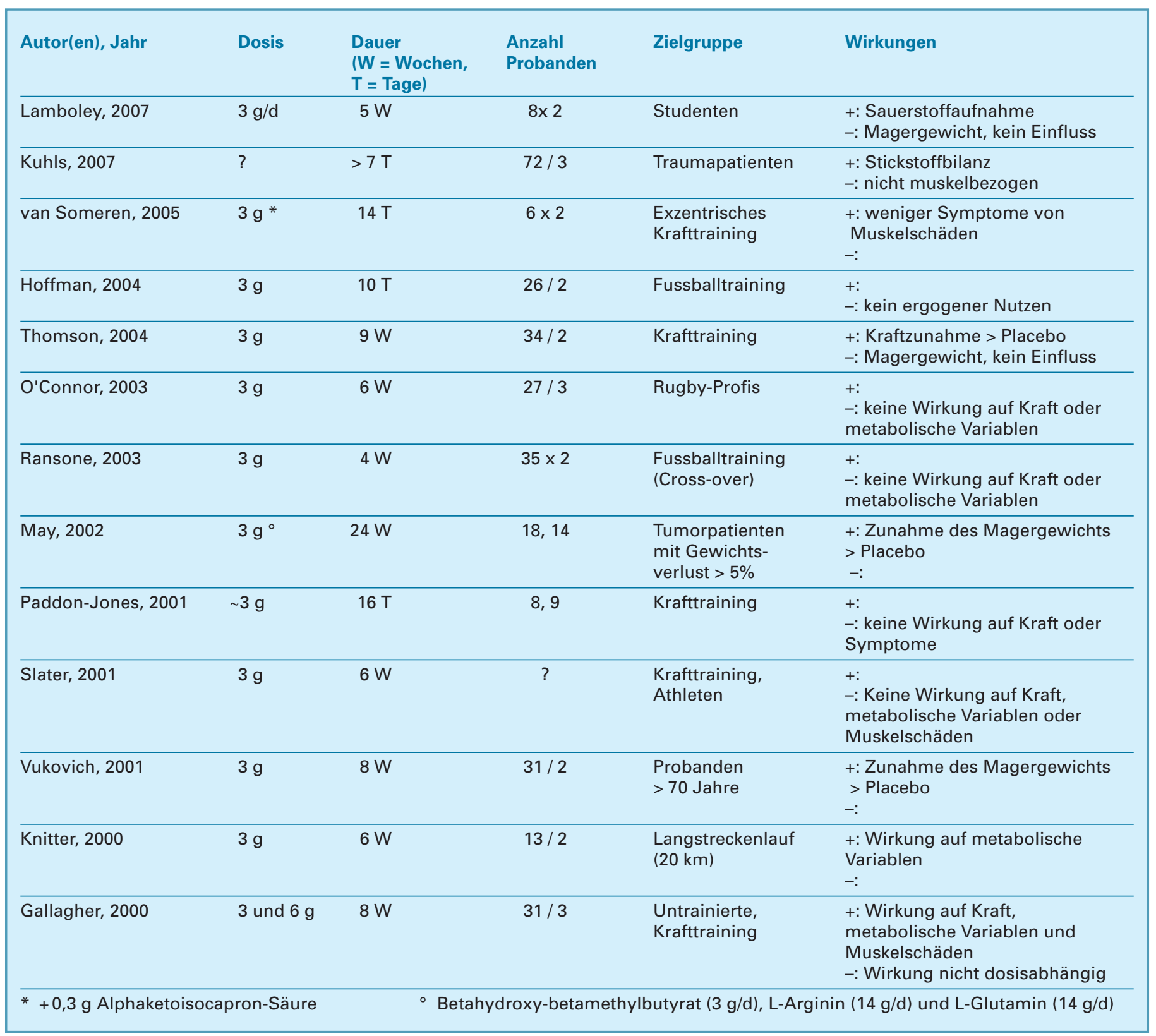

Mittelkettige Triglyzeride (MCT) sind Fette, die kürzere Fettsäuren enthalten als üblich in der Ernährung (Kapronsäure (C6), Laurinsäure (C12) usw.). Diese gelangen vom Darm direkt in die Pfortader und die Leber; sie werden bevorzugt aufgespalten und stellen eine rasch verfügbare Energiequelle dar.

In zwei Studien wurde die Wirkung von MCT geprüft, indem eine Belastung von drei Stunden Dauer unter einer Supplementierung mit $10 \mathrm{~g}$ MCT pro Stunde erfolgte. Es zeigte sich, dass die MCT nur unwesentlich - etwa $5 \%$ - zur Bereitstellung von Energie beitrugen [81,82]. In einer Studie, in der die Teilnehmer $100 \mathrm{~km}$ auf dem Fahrradergometer fahren mussten, wurde die Leistung durch die zusätzliche Gabe von MTC nicht weiter verbessert [83]. Andere Autoren berichten sogar über eine Verschlechterung der Leistung bei der Einnahme von MCT [84]. Grössere Mengen MCT (ab etwa $30 \mathrm{~g}$ ) verursachen zudem oft Magen-Darm-Beschwerden und sind geschmacklich problematisch.

Eine Zusammenfassung über die Bedeutung leistungsfördernder Hilfsstoffe ist in Tabelle 4 dargestellt.

\section{Vitamine}

Eine Zusammenfassung über leistungssteigernden Wirkungen von Vitaminen ist, gemäss einer Publikation aus 2006 [85], in Tabelle 5 dargestellt.

\section{Essstörungen und Sport}

In der Normalbevölkerung haben 2$5 \%$ der weiblichen Bevölkerung Essstörungen. Bei Sportlerinnen, die gewisse Sportarten ausführen, leiden bis $\mathrm{zu}$ über $50 \%$ an Essstörungen [89]. In einer entsprechenden Studie mit Col- 
Tab. 4. Bedeutung leistungsfördernder Hilfsstoffe

\begin{tabular}{|c|c|c|c|}
\hline Hilfsstoff & Leistung bei Mangel an & $\begin{array}{l}\text { Wirkung auf } \\
\text { entsprechendem Stoff }\end{array}$ & $\begin{array}{l}\text { Leistungsfördernde } \\
\text { Muskel-Aufbau Wirkung }\end{array}$ \\
\hline $\begin{array}{l}\text { Puffersubstanzen } \\
\text { (Natriumbikarbonat) }\end{array}$ & --- & Unwirksam & $\begin{array}{l}++ \\
\text { (kurze Wirkung) }\end{array}$ \\
\hline Kreatin & --- & + & $\begin{array}{l}++ \\
\text { (bei spezifischen Gruppen) }\end{array}$ \\
\hline Hydroxymethylbutyrat & --- & Keine Angaben & $\begin{array}{l}+ \\
\text { (bei untrainierten Personen) }\end{array}$ \\
\hline Mittelkettige Triglyzeride & --- & Keine Angaben & Unwirksam \\
\hline
\end{tabular}

Tab. 5. Leistungssteigernde Wirkungen von Vitaminen [85]

\begin{tabular}{|c|c|c|}
\hline Vitamin(e) & Leistungssteigerung & Andere Wirkungen beim Sport \\
\hline Multivitamine & Gering / Wirkungslos & Körpermasse bleibt gleich \\
\hline \multirow[t]{2}{*}{ Vitamine $\mathrm{C}$ und $\mathrm{E}$} & Wirkungslos & Vitamin C: Atemwegsinfekte nehmen ab \\
\hline & & $\begin{array}{l}\text { Vitamine C und E: Effekte des Ozons auf die Lungen nehmen ab [86], } \\
\text { Atemwegsinfekte nehmen eher zu (?) [87] }\end{array}$ \\
\hline Thiamin & Wirkungslos & $\begin{array}{l}\text { Bei erhöhtem Kohlenhydratkonsum und physischer Aktivität } \\
\text { steigt der Thiaminbedarf [88] }\end{array}$ \\
\hline Riboflavin & Keine Angaben & $\begin{array}{l}\text { Bei erhöhtem Kohlenhydratkonsum steigt wahrscheinlich } \\
\text { der Riboflavinbedarf }\end{array}$ \\
\hline Niacin & Wirkungslos & Cholesterinwerte und freie Fettsäuen nehmen ab \\
\hline Pyridoxin & Wirkungslos & Bei erhöhter physischer Aktivität steigt der Pyridoxinbedarf \\
\hline Folat & Wirkungslos & Senkung der Homocysteinwerte (?) \\
\hline Cyanocobalamin & Wirkungslos & Verbesserte Koordination in einer Studie \\
\hline Pantothenat, Biotin & Keine Angaben & Keine Angaben \\
\hline Vitamin A & Wirkungslos & $\begin{array}{l}\text { Eventuell besteht ein erhöhter Bedarf; } \\
\text { Achtung: Vitamin A ist in grösseren Mengen toxisch! }\end{array}$ \\
\hline Vitamine $\mathrm{D}$ und $\mathrm{K}$ & Keine Angaben / Wirkungslos & Keine Angaben / Wirkungslos \\
\hline
\end{tabular}

lege-Athletinnen ergab sich, dass $62 \%$ der Sportlerinnen mehr als 2,5 kg Gewicht abnehmen wollten; nur 15\% ernährten sich richtig [90]. Ein erhöhtes Risiko für eine Essstörung oder die sogenannte „female athlete triad” (Kombination von Essstörung, Ausbleiben der Menstruation und Osteoporose) besteht bei ästhetischen Sportarten (Kunstturnen, Eiskunstlaufen), Ausdauersportarten, Sportarten mit Gewichtsklassen (Judo, Ringen etc.) sowie bei Klettern oder Skispringen.

Die Essstörungen dieser jungen Frauen - in seltenen Fällen auch Männer - sind äusserst schwierig zu diagnostizieren. Häufig wird die Erkrankung verschwiegen oder verneint. Wird sie nicht behandelt, führt sie zu einer oft schon im Jugendalter massiven Osteoporose, besonders bei Mäd- chen, bei denen aufgrund der Essstörung die Menstruation ausbleibt [91]. Auf der Website von Swiss Olympic (www.swissolympic.ch) findet sich ein Fragebogen zur Erfassung der „female athlete triad".

Leistungseinbrüche oder Episoden von Müdigkeit können durch eine Eisenmangelanämie oder einen latenten Eisenmangel ausgelöst werden. Beide Erscheinungen sind unter jugendlichen Sportlern sehr häufig, insbesondere bei Mädchen, die menstruieren. Ein weiterer Risikofaktor ist eine vegetarische Ernährung.

\section{Schlussfolgerungen}

- Damit ein Leistungszuwachs erzielt werden kann, muss ein Training nach dem Prinzip der schrittweise steigenden Belastung aufgebaut sein. Die Ernährung von Sportlerinnen und Sportlern sollte auf der Lebensmittelpyramide aufbauen. Die von Sportlern zusätzlich benötigten Nährstoffmengen sind unter anderem in Infoblättern der ETH Zürich vermerkt.

- Die körperliche Leistungsfähigkeit nimmt zwar mit dem Alter ab, die Leistungsfähigkeit kann aber auch im Alter durch Training erhalten und verbessert werden. Die positive Wirkung des Trainings auf die Blutfettwerte und besonders auf Herz und Kreislauf ist abhängig von der Trainingsintensität.

- Schwitzen ist ein zentraler Regulationsmechanismus für die Körpertemperatur. Mit dem Schweiss 
werden neben dem Wasser auch Elektrolyte ausgeschieden. Diese müssen wieder ersetzt werden.

- Eine Supplementierung von Magnesium verbessert wohl nur dann die Leistung, wenn ein Magnesiummangel vorliegt. Dasselbe dürfte auch für Zink zutreffen. Prospektive Daten fehlen.

- Eine Supplementierung von Eisen ist beim Vorliegen eines Eisenmangels angebracht (Ausdauersportarten, Frauen).

- Für die Supplemente Chrom, Selen, Mangan, Vanadium oder Bor sowie für mittelkettige Triglyzeride oder Vitamine sind keine sportspezifischen Empfehlungen möglich.

- Leistungsfördernde Wirkstoffe sind Kreatin (Krafttraining) und wahrscheinlich Hydroxymethylbutyrat (bei untrainierten Personen); die Zunahme der Magermasse sowie der Muskelkraft beträgt ca. 1\%/Woche.

- Die einmalige oder wiederholte kurzzeitige Anwendung von Puffersubstanzen wie Natriumbikarbonat oder Natriumcitrat hat ein anerkanntes leistungsförderndes Potential (Klasse-A-Supplement). Allerdings sind als Nebenwirkungen häufig Magen-Darm-Probleme und gelegentlich auch Alkalosen beschrieben worden.

- Speziell bei jugendlichen Sportlerinnen ist auf Essstörungen zu achten, besonders bei ästhetischen und Ausdauersportarten sowie bei Sportarten mit Gewichtsklassen.

This paper is an updated summary of an expert-workshop 2007 sponsored by BIOMED, Dübendorf, Switzerland.

\section{Literatur}

1 Martin BW, Sonja Kahlmeier, Francesca Racioppi, Finn Berggren, Mari Miettinen, Jean-Michel Oppert, Harry Rutter, Radim-lachta, Mireille van Poppel, Jozica Maucec Zakotnik, Dirk Meusel, Pekka Oja, Michael Sjöström: Evidence-based physical activity promotion - HEPA Europe, the European Network for the Promotion of HealthEnhancing Physical Activity. J Public Health 2006; 14: 53-57. DOl 10.1007/s10389-006-0029- 5.

2 Andersen JL, Peter Schjerling und Bengt Saltin. Muskeln, Gene und Leistungssport SPEKTRUM DER WISSENSCHAFT. März 2001, 70-75.

3 Haman F, Stéphane R. Legault and Jean-Michel Weber. Fuel selection during intense shivering in humans: EMG pattern reflects carbohydrate oxidation. J Physiol. 2004 Apr 1; 556(Pt 1): 305-13.

4 Zierath JR, Hawley JA: Skeletal Muscle Fiber
Type: Influence on Contractile and Metabolic Properties. PLoS Biology Vol. 2, No. 10, e348 doi:10.1371/journal. pbio.0020348.

5 Kirwan J.P. and L.F. del Aguila. Insulin signalling, exercise and cellular integrity. Biochem. Soc. Trans. (2003) 31, 1281-128).

6 Mannhart C, Paolo Colombani: Grundlagen de Sporternährung - die elementare Bedeutung de Energie-, Makronährstoff- und Flüssigkeitszufuhr. Schweizerische Zeitschrift für Sportmedizin und Sporttraumatologie 2001; 49 (3): 125-130.

7 Maughan RJ. Nutritional status, metabolic responses to exercise and implications for performance. Biochem. Soc. Trans. 2003; 31: 1267-126.

8 Wolfarth B. Genetische Polymorphismen be hochtrainierten Ausdauerathleten - die Genathlete-Studie. Deutsche Zeitschrift für Sportmedizin 2002; 53 (12): 338-344.

9 Montgomery HE, Marshall R, Hemingway $\mathrm{H}$ Myerson S, Clarkson P, Dollery C, Hayward M Holliman DE, Jubb M, World M, Thomas EL, Brynes AE, Saeed N, Barnard M, Bell JD, Prasad K, Rayson M, Talmud, PJ, Humphries SE: Human gene for physical performance [letter]. Nature 393 (1998) 221-222.

10 Weston AR, O. Karamizrak1, A. Smith, T. D Noakes, and Kathryn H. Myburgh. African runners exhibit greater fatigue resistance, lower lactate accumulation, and higher oxidative enzyme activity. J Appl Physiol 86: 915-923, 1999.

11 McNally EM. Powerful Genes - Myostatin Regulation of Human Muscle Mass. NEJM June 24 2004 Volume 350 (26): 2642-2644.

12 Fitzgerald MD, Tanaka H, Tran ZV, Seals DR. Agerelated declines in maximal aerobic capacity in regularly exercising vs. sedentary women: meta-analysis. J Appl Physiol. 1997; 83(1): 160-5.

13 Mora S, Rita F. Redberg, Yadong Cui, Maura K. Whiteman, Jodi A. Flaws, A. Richey Sharrett, Roger S. Blumenthal: Ability of Exercise Testing to Predict Cardiovascular and All-Cause Death in Asymptomatic Women. A 20-Year Follow-up of the Lipid Research Clinics Prevalence Study. JAMA. 2003; 290: 1600-1607.

14 Okamoto K: Life expectancy at the age of 65 years and environmental factors: An ecological study in Japan. Archives of Gerontology and Geriatrics 2006; 43(1): 85-91 doi:10.1016/ j.archger. 2005.09.005.

15 Kraus WE, Joseph A. Houmard, Brian D. Duscha, Kenneth J. Knetzger, Michelle B. Wharton, Jennifer S. McCartney, Connie W. Bales, Sarah Henes, Gregory. Samsa P, James D. Otvos, Krishnaji R. Kulkarni, and Cris A. Slentz: Effects of the Amount and Intensity of Exercise on Plasma Lipoproteins NEJM 2002; 347(19): 1483-1492.

16 Duncan GE, Stephen D. Anton, Sumner J. Sydeman, Robert L. Newton, Jr, Joyce A. Corsica Patricia E. Durning, Timothy U. Ketterson, A Daniel Martin, Marian C. Limacher, Michael G. Perri: Prescribing Exercise at Varied Levels of Intensity and Frequency. A Randomized Trial. Arch Intern Med. 2005: 165: 2362-2369.

17 Roberts CK and R. James Barnard: Effects of exercise and diet on chronic disease. J Appl Physiol 98: 3-30, 2005; doi: 10.1152/japplphysiol. $00852.20048750-7587 / 05$

18 Snowling NJ and Will G. Hopkins: Effects of Dif ferent Modes of Exercise Training on Glucose Control and Risk Factors for Complications in Type 2 Diabetic Patients. A meta-analysis. Diabetes Care 29: 2518-2527, 2006 DOI: 10.2337/ dc06-1317.

19 JOINT POSITION STATEMENT, AHA/ACSM Joint Statement: Recommendations for Cardiovascular Screening, Staffing, and Emergency Policies at Health/Fitness Facilities. June 1998 Volume 30, Number 6 www.ms-se.com/pt/pt-core/templatejournal/msse/ media/0698c.htm.

20 Casa DJ, Priscilla M. Clarkson, \& William O. Roberts: American College of Sports Medicine Roundtable on Hydration and Physical Activity: Consensus Statements. Current Sports Medicine Reports 2005, 4: 115-127.
21 Ronge R. Marathonläufer: Wem droht eine Hyponatriämie? Dtsch Med Wochenschr 2005; 130 Nr. 27.

22 Almond CSD et al.: Hyponatremia among Runners in the Boston Marathon: N Engl J Med 2005; 352: 1550-1556.

23 POSITION STAND Exercise and Fluid Replacement. Medicine \& Science in Sports \& Exercise, February 1, 2007 DOI: 10.1249/mss.0b013e31 802 ca597.

24 Shirreffs SM, Maughan RJ: Whole body sweat collection in humans: an improved method with preliminary data on electrolyte content. J Appl Physiol. 1997 Jan; 82(1): 336-41.

25 Maughan RJ. Fluid and electrolyte loss and replacement in exercise. In: Oxford Textbook of Sports Medicine, edited by M. Harries, C Williams, W. D. Stanish, and L. J. Micheli. Oxford, UK: Oxford Univ. Press, 1994, p. 82-93.

26 Wenk C, M. Kuhnt, P. Kunz und G. Steiner: Methodische Untersuchungen zur Schätzung des Verlustes von Natrium, Kalium, Calcium und Magnesium über den Schweiss am Beispiel eines 10-km-Laufes. Zeitschrift für Ernährungswissenschaft. 1993, 32: 301-307.

27 Verde T, R. J. Shephard, P. Corey and R. Moore: Sweat composition in exercise and in heat. J Appl Physiol 53: 1540-1545, 1982.

28 Patterson MJ Stuart D. R. Galloway and Myra A. Nimmo: Variations in regional sweat composition in normal human males. Experimental Physiology (2000) 85.6, 869-87.

29 Buchman AL, Keen C, Commisso J, Killip D, Ou CN, Rognerud CL, Dennis K, Dunn JK: The effect of a marathon run on plasma and urine mineral and metal concentrations. J Am Coll Nutr (1998 Apr) 17(2): 124-7.

30 Kay BF, Bailey, SM: Original Research Geographical Variations in Heart Deaths and Diabetes: Effect of Climate and a Possible Relationship to Magnesium. Journal of the American College of Nutrition, Vol. 23, No. 5, 521S-524S (2004).

31 Lukaski HC and Forrest H. Nielsen: Human Nutrition and Metabolism. Dietary Magnesium Depletion Affects Metabolic Responses during Submaximal Exercise in Postmenopausal Women. J. Nutr. 132: 930-935, 2002.

32 Brilla L.R. and V. Conte: Effects of a novel zincmagnesium formulation on hormones and strength. J Exerc Physiol Online, 2000. 3(4): p. www.css.edu/users/tboone2/asep/ BrillaV2.pdf

33 Zimmermann MB: Vitamin and mineral supplementation and exercise performance. Schweizerische Zeitschrift für "Sportmedizin und Sporttraumatologie» 51 (1), 53-57, 2003.

34 Finstad E W, Newhouse I J, Lukaski H C, Mcauliffe J E and Stewart C R: The effects of magnesium supplementation on exercise performance. Med. Sci. Sports Exerc., Vol. 33, No. 3, 2001, pp. 493-498.

35 Lukaski HC: Magnesium, zinc, and chromium nutrition and athletic performance. Can J Appl Physiol (2001) 26 Suppl: S13-22.

36 Van Loan MD, Sutherland B, Lowe NM, Turnlund JR, King JC: The effects of zinc depletion on peak force and total work of knee and shoulder extensor and flexor muscles. Int J Sport Nutr 1999; 9(2): 125-35.

37 Cordova A, Navas FJ: Effect of training on zinc metabolism: changes in serum and sweat zinc concentrations in sportsmen. Ann Nutr Metab (1998) 42(5): 274-82.

38 Kikukawa A, Kobayashi A: Changes in urinary zinc and copper with strenuous physical exercise. Aviat Space Environ Med 2002; 73(10): 991-5.

39 Mundie TG, Hare B: Effects of resistance exercise on plasma, erythrocyte, and urine $\mathrm{Zn}$. Biol Trace Elem Res 2001; 79(1): 23-8.

40 DeRuisseau KC, Cheuvront SN, Haymes EM, Sharp RG: Sweat iron and zinc losses during prolonged exercise. Int J Sport Nutr Exerc Metab 2002; 12(4): 428-37. 
41 Horn S, M.D. and Edward Feller, M.D: Gastrointestinal Bleeding in Endurance Runners. AMAA Journal/Winter 2003, 16 (1): 5-6.

42 Fallon KE: Utility of hematological and iron-related screening in elite athletes. Clin J Sport Med (2004 May) 14(3): 145-52

43 Brownlie T, Utermohlen V, Hinton PS, Haas JD: Tissue iron deficiency without anemia impairs adaptation in endurance capacity after aerobic training in previously untrained women. Am J Clin Nutr (2004 Mar) 79(3): 437-43.

44 Committee on Sports Medicine and Fitness. Intensive Training and Sports Specialization in Young Athletes. Pediatrics 2000; 106; 154-157 DOI: 10.1542/peds.106.1.154

45 Zimmermann MB. Vitamin and mineral supplementation and exercise performance. Schweizerische Zeitschrift für "Sportmedizin und Sporttraumatologie» 51 (1), 53-57, 2003.

46 Brownlie T, Utermohlen V, Hinton PS, Haas JD: Tissue iron deficiency without anemia impairs adaptation in endurance capacity after aerobic training in previously untrained women. Am J Clin Nutr (2004 Mar) 79(3): 437-43.

47 Brutsaert TD, Hernandez-Cordero S, Rivera J, Viola T, Hughes G, Haas JD: Iron supplementation improves progressive fatigue resistance during dynamic knee extensor exercise in iron-depleted, nonanemic women. Am J Clin Nutr (2003 Feb) 77(2): 441-8.

$48 \mathrm{Zhu} \mathrm{YI}$, Haas JD: Altered metabolic response of iron-depleted nonanemic women during a $15-\mathrm{km}$ time trial. J Appl Physiol (1998 May) 84(5) 1768-75.

49 Walker L.S., et al., Chromium picolinate effects on body composition and muscular performance in wrestlers. Med Sci Sports Exerc 1998; 30(12): 1730-7

50 Volek JS, Silvestre R, Kirwan JP, Sharman MJ, Judelson DA, Spiering BA, Vingren JL, Maresh CM, Kraemer WJ (2006): Effects of chromium supplementation on glycogen synthesis after high-intensity exercise, Med. Sci. Sports Exerc., 38(12): 2102-2109, December 2006.

51 Vincent J.B: The Potential Value and Toxicity of Chromium Picolinate as a Nutritional Supplement Weight Loss Agent and Muscle Development Agent. Sports Medicine, Volume 33, Number 3, 2003, pp. 213-230(18).

52 Margaritis I, Tessier F, Prou E, Marconnet P, Marini JF: Effects of endurance training on skeletal muscle oxidative capacities with and without selenium supplementation. J Trace Elem Med Biol (1997 Apr) 11(1): 37-43.

53 Fawcett J.P., et al., The effect of oral vanadyl sulfate on body composition and performance in weight-training athletes. Int J Sport Nutr, 1996. 6(4): p. 382-90

54 Fawcett J.P. et al. Oral vanadyl sulphate does not affect blood cells, viscosity or biochemistry in humans. Pharmacol Toxicol 1997; 80(4): p. 202-6.

55 Green N.R. and A.A. Ferrando: Plasma boron and the effects of boron supplementation in males. Environ Health Perspect 1994; 102 Suppl 7: 73-7.

56 Ferrando A.A. and N.R. Green: The effect of boron supplementation on lean body mass, plasma testosterone levels, and strength in male bodybuilders. Int J Sport Nutr 1993; 3(2): 140-9.

57 Meacham SL, Taper LJ, Volpe SL: Effects of boron supplementation on bone mineral density and dietary, blood, and urinary calcium, phosphorus, magnesium, and boron in female athletes. Environ Health Perspect 1994; 102, Suppl 7: 79-82.

58 Aoi W, Yuji Naito, Toshikazu Yoshikawa: Exercise and functional foods. Nutrition Journal 2006, 5: 15 (5 June 2006) (doi: 10.1186/1475-2891-5-15.).

59 Kreider RB, Anthony L. Almada, Jose Antonio Craig Broeder, Conrad Earnest, Mike Greenwood, Thomas Incledon, Douglas S. Kalman, Susan M. Kleiner, Brian Leutholtz, Lonnie M. Lowery, Ron Mendel, Jeffrey R. Stout, Darryn S. Willoughby, Tim N. Ziegenfuss. ISSN Exercise \& Sport Nutrition Review: Research \& Recommendations.
Sports Nutrition Review Journal 2004; 1 (1): 1-44. (www.sportsnutritionsociety.org).

60 Nissen SL and Sharp RL: Effect of dietary supplements on lean mass and strength gains with resistance exercise: a meta-analysis. J Appl Physiol 94: 651-659, 2003

61 Bishop D, Claudius B: Effects of induced metabolic alkalosis on prolonged intermittent-sprin performance. Med Sci Sports Exerc. 2005 May; 37(5): 759-67.

62 Price M, Moss P, Rance S. Effects of sodium bicarbonate ingestion on prolonged intermittent exercise. Med Sci Sports Exerc. 2003; 35(8): 1303-8.

63 Matson LG, Tran ZV. Effects of sodium bicarbonate ingestion on anaerobic performance: a metaanalytic review. Int J Sport Nutr. 1993; 3(1): 2-28.

64 Van Montfoort MC, Van Dieren L, Hopkins WG, Shearman JP. Effects of ingestion of bicarbonate, citrate, lactate, and chloride on sprint running. Med Sci Sports Exerc. 2004 Jul; 36(7): 1239-43.

65 American college of sports medicine: The physiological and health affects of oral creatine supplementation. Med. Sci. Sports Exerc. Vol.32, No3 pp 706-717, 2000.

66 Dempsey RL, Michael F. Mazzone, LInda N. Meurer: Does oral creatine supplementation improve strength? A meta-analysis. J Fam Pract 2002; 51: 945-952.

67 Branch JD: Effect of creatine supplementation on body composition and performance: a metaanalysis. Int J Sport Nutr Exerc Metab. 2003 13(2): 198-226.

68 Lamboley CR, Royer D, Dionne IJ: Effects of beta-hydroxy-beta-methylbutyrate on aerobic-performance components and body composition in college students. In: Int J Sport Nutr Exerc Metab (2007 Feb) 17(1): 56-69.

69 Kuhls DA, Rathmacher JA, Musngi MD, Frisch DA, Nielson J, Barber A, Maclntyre AD, Coates JE, Fildes JJ: Beta-hydroxy-beta-methylbutyrate supplementation in critically ill trauma patients. $J$ Trauma 2007; 62(1): 125-31; discussion 131-2.

70 Van Someren KA, Edwards AJ, Howatson G: Supplementation with beta-hydroxy-beta-methylbutyrate (HMB) and alpha- ketoi-socaproic acid (KIC) reduces signs and symptoms of exercise-induced muscle damage in man. In: Int J Sport Nutr Exerc Metab (2005 Aug) 15(4): 413-24.

71 Hoffman JR, Cooper J, Wendell M, Im J, Kang J: Effects of beta-hydroxy beta-methylbutyrate on power performance and indices of muscle damage and stress during high-intensity training. In: Strength Cond Res (2004 Nov) 18(4): 747-52

72 Thomson JS: beta-Hydroxy-beta-Methylbutyrate (HMB) supplementation of resistance trained men. Asia Pac J Clin Nutr (2004) 13 (Suppl): S59.

73 O'Connor DM, Crowe MJ: Effects of betahydroxy-beta-methylbutyrate and creatine monohydrate supplementation on the aerobic and anaerobic capacity of highly trained athletes. $J$ Sports Med Phys Fitness (2003 Mar) 43(1): 64-8.

74 Ransone J, Neighbors K Lefavi R, Chromiak J: The effect of beta-hydroxy beta-methylbutyrate on muscular strength and body composition in collegiate football players. In: J Strength Cond Res (2003 Feb) 17(1): 34-9.

75 May PE, Barber A, D'Olimpio JT, Hourihane A Abumrad NN: Reversal of cancer-related wasting using oral supplementation with a combination of beta-hydroxy-beta-methylbutyrate, arginine, and glutamine. Am J Surg (2002 Apr) 183(4): 471-9.

76 Paddon-Jones D, Keech A, Jenkins D: Short-term beta-hydroxy-beta-methylbutyrate supplementation does not reduce symptoms of eccentric muscle damage. In: Int J Sport Nutr Exerc Metab (2001 Dec) 11(4): 442-50.

77 Slater G, Jenkins D, Logan P, Lee H, Vukovich M Rathmacher JA, Hahn AG: Beta-hydroxy-betamethylbutyrate (HMB) supplementation does no affect changes in strength or body composition during resistance training in trained men. In: Int $J$ Sport Nutr Exerc Metab 2001; 11(3): 384-96.
78 Vukovich MD, Stubbs NB, Bohlken RM: Body composition in 70-year-old adults responds to dietary beta-hydroxy-beta-methylbutyrate similarly to that of young adults. In: J Nutr (2001 Jul) 131(7): 2049-52.

79 Knitter AE, Panton L, Rathmacher JA, Petersen A, Sharp R: Effects of beta-hydroxy-beta-methylbutyrate on muscle damage after a prolonged run. In: J Appl Physiol (2000 Oct) 89(4): 1340-4.

80 Gallagher PM, Carrithers JA, Godard MP, Schulze KE, Trappe SW: Beta-hydroxy-beta-methylbutyrate ingestion, Part I: effects on strength and fat free mass. In: Med Sci Sports Exerc (2000 Dec) 32(12): 2109-15.

81 Jeukendrup AE, Saris WH, Schrauwen P, Brouns F, Wagenmakers AJ: Metabolic availability of medium-chain triglycerides coingested with carbohydrates during prolonged exercise. J Appl Physiol 79: 756-62, 1995

82 Vistisen B, Lars Nybo, Xuebing Xu, Carl-Erik Høy, and Bente Kiens: Minor amounts of plasma medium-chain fatty acids and no improved time trial performance after consuming lipids. J Appl Physiol 95: 2434-2443, 2003

83 Angus DJ, Mark Hargreaves, Jane Dancey, and Mark A. Febbraio: Effect of carbohydrate or carbohydrate plus medium-chain triglyceride ingestion on cycling time trial performance. J Appl Physiol 88: 113-119, 2000

84 Thorburn MS, Bodil Vistisen, Rhys M. Thorp, Mike J. Rockell, Asker E. Jeukendrup, Xuebing $\mathrm{Xu}$, and David S. Rowlands: Attenuated gastric distress but no benefit to performance with adaptation to octanoate-rich esterified oils in welltrained male cyclists. J Appl Physiol 2006; 101: 1733-1743.

85 Zusammenfassung aus Sports Nutrition - Vitamin and Trace Elements - Ed. Judy A. Driskell \& Ira Wolinsky. Taylor \& Francis, 2006

86 Grievink L, Zijlstra AG, Ke X, Brunekreef B: Double-blind intervention trial on modulation of ozone effects on pulmonary function by antioxidant supplements. Am J Epidemiol 1999; 149(4): 306-14.

87 Hemila H, Virtamo J, Albanes D, Kaprio J: Physical activity and the common cold in men administered vitamin $\mathrm{E}$ and beta-carotene. Med Sci Sports Exerc (2003 Nov) 35(11): 1815-20.

88 Manore MM: Effect of physical activity on thiamine, riboflavin, and vitamin B-6 requirements. Am J Clin Nutr 2000: 72 (2 Suppl): 598S-606S.

89 Kriemler S, Sportmedizin: Untersuchungsvorschläge für verschiedene Sportarten. Pediatrica 2005; Vol. 16: No. 4, 34-36.

90 Hinton PS, Sanford TC, Davidson MM, Yakushko OF, Beck NC: Nutrient intakes and dietary behaviors of male and female collegiate athletes.Int J Sport Nutr Exerc Metab 2004; 14(4): 389-405.

91 Birch K: Clinical review. ABC of sports and exercise medicine Female athlete triad. BMJ 2005; 330: 244-246 (29 January), doi: 10.1136/bmj.330. 7485.244

\section{Korrespondenzadresse:}

Prof. Dr. med. Reinhard Saller

UniversitätsSpital Zürich

Dep. für Innere Medizin

Institut für Naturheilkunde

Rämistrasse 100, CH-8091 Zürich

reinhard.saller@usz.ch 